\title{
Simplified realization of two-qubit quantum phase gate with four-level systems in cavity QED
}

\author{
Chui-Ping Yang, ${ }^{1,2}$ Shih-I Chu, ${ }^{2}$ and Siyuan Han ${ }^{1}$ \\ ${ }^{1}$ Department of Physics and Astronomy, University of Kansas, Lawrence, Kansas 66045, USA \\ ${ }^{2}$ Department of Chemistry, University of Kansas, and Kansas Center for Advanced Scientific Computing, \\ Lawrence, Kansas 66045, USA
}

(Received 24 November 2003; revised manuscript received 18 May 2004; published 14 October 2004)

\begin{abstract}
We propose a method for realizing two-qubit quantum phase gate with 4-level systems in cavity QED. In this proposal, the two logical states of a qubit are represented by the two lowest levels of each system, and two intermediate levels of each system are utilized to facilitate coherent control and manipulation of quantum states of the qubits. The present method does not involve cavity-photon population during the operation. In addition, we show that the gate can be achieved using only two-step operations.
\end{abstract}

DOI: 10.1103/PhysRevA.70.044303

PACS number(s): 03.67.Lx, 03.65.-w

Recently, a number of schemes have been proposed for realizing quantum computation with up to 6-level systems based on cavity QED or trap assistance [1-10]. These schemes play an important role in building up quantum computers. In this note, we wish to present an alternative method for achieving a two-qubit quantum phase gate with two 4-level systems in cavity QED (a similar model was previously considered in Refs. [9]). As is well known, a two-qubit phase gate with single-qubit rotations makes a set of operation, which is universal for quantum computing. The present scheme operates essentially by having the pulse, applied to one system, to couple to the transition between the level $\left|g^{\prime}\right\rangle$ and the intermediate level $|a\rangle$, while having the pulse, applied to the other system, to couple to the transition between the level $\left|g^{\prime}\right\rangle$ and the other intermediate level $|b\rangle$ (Fig. 1). As shown below, as well as the cavity mode being only virtually excited during the operation, the gate can be achieved using only two steps (a two-qubit joint operation plus a singlequbit operation). Therefore, the present operation is significantly simplified.

Consider two individual systems I and II each having four levels $|g\rangle,\left|g^{\prime}\right\rangle,|a\rangle$, and $|b\rangle$ with energy eigenvalues $E_{g}, E_{g^{\prime}}$, $E_{a}$, and $E_{b}$, respectively (Fig. 1). The transition between the two lowest levels $|g\rangle$ and $\left|g^{\prime}\right\rangle$ is assumed to be forbidden or very weak. Suppose that the cavity mode is coupled to the $|a\rangle \leftrightarrow|b\rangle$ transition of each system and that pulse I (II) is coupled to the $\left|g^{\prime}\right\rangle \leftrightarrow|b\rangle\left(\left|g^{\prime}\right\rangle \leftrightarrow|a\rangle\right)$ transition of system I (II) (Fig. 1). The Hamiltonian for the whole system in the Schrödinger picture can then be written as

$$
\begin{aligned}
H= & \sum_{l=\mathrm{I}, \mathrm{II}} \sum_{k} E_{k} \sigma_{k k l}+\hbar \omega_{c} c^{\dagger} c+\sum_{l=\mathrm{I}, \mathrm{II}} \hbar\left(\mu c^{\dagger} \sigma_{a b l}+\text { H.c. }\right) \\
& +\hbar\left(\Omega_{\mathrm{I}} e^{i \omega_{\mathrm{I}} t} \sigma_{g^{\prime} b \mathrm{I}}+\Omega_{\mathrm{II}} e^{i \omega_{\mathrm{II}} t} \sigma_{g^{\prime} a \mathrm{II}}+\text { H.c. }\right),
\end{aligned}
$$

where subscripts I and II represent systems I and II as well as pulses I and II, $c^{\dagger}$ and $c$ are the photon creation and annihilation operators of the cavity mode with frequency $\omega_{c} ; \mu$ is the coupling constant between the cavity mode and the $|a\rangle \leftrightarrow|b\rangle$ transition of each system; $\Omega_{\mathrm{I}}$ is the Rabi frequency of pulse I for the two levels $\left|g^{\prime}\right\rangle$ and $|b\rangle$ of system I, while $\Omega_{\mathrm{II}}$ is the Rabi frequency of pulse II for the two levels $\left|g^{\prime}\right\rangle$ and $|a\rangle$ of system II; $\omega_{\mathrm{I}}$ and $\omega_{\mathrm{II}}$ are carrier frequencies of the two pulses I and II, respectively; $\sigma_{k k l}=|k\rangle_{l}\langle k|\left(k=g, g^{\prime}, a, b\right)$, $\sigma_{g^{\prime} b l}=\left|g^{\prime}\right\rangle_{l}\langle b|$ and $\sigma_{g^{\prime} a l}=\left|g^{\prime}\right\rangle_{l}\langle a|$.

Suppose that the cavity mode is largely detuned from the $|a\rangle \leftrightarrow|b\rangle$ transition, i.e., $\Delta_{c}=\omega_{b a}-\omega_{c} \gg \mu$, and pulse I is largely detuned from the $\left|g^{\prime}\right\rangle \leftrightarrow|b\rangle$ transition of system I, i.e., $\Delta_{\mathrm{I}}=\omega_{b g^{\prime}}-\omega_{\mathrm{I}} \gg \Omega_{\mathrm{I}}$ while pulse II is largely detuned from the $\left|g^{\prime}\right\rangle \leftrightarrow|a\rangle$ transition of system II, i.e., $\Delta_{\mathrm{II}}=\omega_{\mathrm{II}}-\omega_{a g^{\prime}} \gg \Omega_{\mathrm{II}}$, where $\omega_{b a}=\left(E_{b}-E_{a}\right) / \hbar, \omega_{b g^{\prime}}=\left(E_{b}-E_{g^{\prime}}\right) / \hbar$, and $\omega_{a g^{\prime}}=\left(E_{a}\right.$ $\left.-E_{g^{\prime}}\right) / \hbar$ (Fig. 1). Under this condition, the level $|b\rangle$ for system I and the level $|a\rangle$ for system II can be adiabatically eliminated [11]. Thus the effective Hamiltonian in the interaction picture can be written as

$$
\begin{aligned}
H_{\mathrm{I}}= & -\hbar \frac{\Omega_{\mathrm{I}}^{2}}{\Delta_{\mathrm{I}}} \sigma_{g^{\prime} g^{\prime} \mathrm{I}}-\hbar \frac{\mu^{2}}{\Delta_{c}} c^{\dagger} c \sigma_{a a \mathrm{I}}+\hbar \frac{\Omega_{\mathrm{II}}^{2}}{\Delta_{\mathrm{II}}} \sigma_{g^{\prime} g^{\prime} \mathrm{II}}+\hbar \frac{\mu^{2}}{\Delta_{c}} c^{\dagger} c \sigma_{b b \mathrm{II}} \\
& -\hbar\left(\chi_{\mathrm{I}} e^{i \delta_{\mathrm{I}} t} \sigma_{g^{\prime} a \mathrm{I}} c+\text { H.c. }\right)+\hbar\left(\chi_{\mathrm{II}} e^{i \delta_{\mathrm{II}} t} \sigma_{g^{\prime} b \mathrm{II}}^{\dagger} c+\text { H.c. }\right),
\end{aligned}
$$

where $\delta_{\mathrm{I}}=\Delta_{\mathrm{I}}-\Delta_{c}, \quad \delta_{\mathrm{II}}=\Delta_{\mathrm{II}}-\Delta_{c}, \quad \chi_{\mathrm{I}}=\left(\Omega_{\mathrm{I}} \mu / 2\right)\left(1 / \Delta_{c}+1 / \Delta_{\mathrm{I}}\right)$ and $\chi_{\mathrm{II}}=\left(\Omega_{\mathrm{II}} \mu / 2\right)\left(1 / \Delta_{c}+1 / \Delta_{\mathrm{II}}\right)$. The first two terms in Eq. (2) are ac-Stark shifts of the levels $\left|g^{\prime}\right\rangle$ and $|a\rangle$ for system I induced by pulse I and the cavity mode, respectively; while the second two terms in Eq. (2) are ac-Stark shifts of the

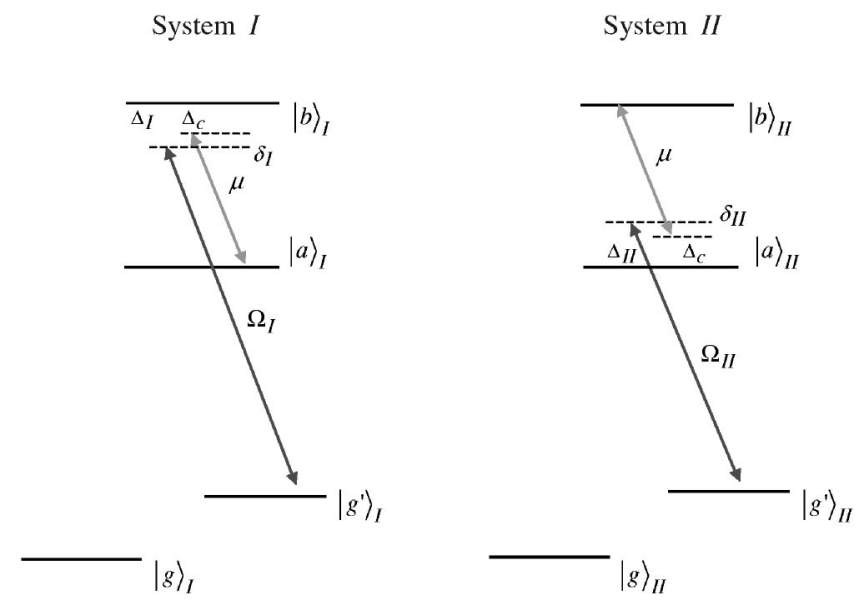

FIG. 1. Level diagram of two systems with four levels $|g\rangle,\left|g^{\prime}\right\rangle$, $|a\rangle$, and $|b\rangle$. 


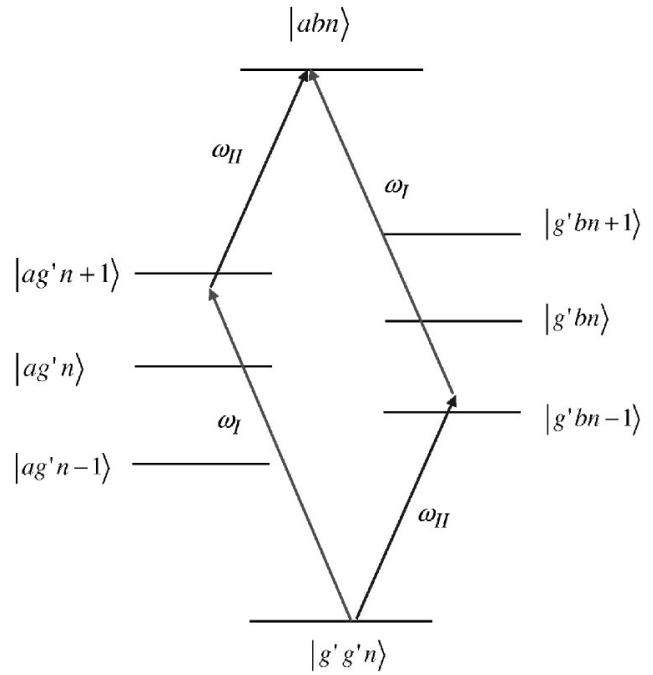

FIG. 2. Illustration of the energy conversing transition between $\left|g^{\prime} g^{\prime} n\right\rangle$ and $|a b n\rangle$ mediated by $\left|a g^{\prime} n+1\right\rangle$ and $\left|g^{\prime} b n-1\right\rangle$.

levels $\left|g^{\prime}\right\rangle$ and $|b\rangle$ for system II induced by pulse II and the cavity mode, respectively. Finally, the last two terms in Eq. (2) are the familiar Jaynes-Cummings interaction, describing the Raman coupling of the two levels $\left|g^{\prime}\right\rangle$ and $|a\rangle$ for system I as well as the Raman coupling of the two levels $\left|g^{\prime}\right\rangle$ and $|b\rangle$ for system II, respectively.

When $\delta_{\mathrm{I}}=\Delta_{\mathrm{I}}-\Delta_{c} \gg \mu^{2} / \Delta_{c}, \Omega_{\mathrm{I}}^{2} / \Delta_{\mathrm{I}}, \chi_{\mathrm{I}} ; \quad$ and $\quad \delta_{\mathrm{II}}=\Delta_{\mathrm{II}}-\Delta_{c}$ $\gg \mu^{2} / \Delta_{c}, \Omega_{\mathrm{II}}^{2} / \Delta_{\mathrm{II}}, \chi_{\mathrm{II}}$, there is no energy exchange between the systems (I, II) and the cavity mode. In the following we set $\delta_{\mathrm{I}}=\delta_{\mathrm{II}}=\delta$ by having $\Delta_{\mathrm{I}}=\Delta_{\mathrm{II}}$ which can be readily realized via adjusting the frequencies of the two pulses (Fig. 1). Thus, the energy conversing transitions are between $\left|g^{\prime} g^{\prime} n\right\rangle$ and $|a b n\rangle$, mediated by $\left|a g^{\prime} n+1\right\rangle$ and $\left|g^{\prime} b n-1\right\rangle$ where the first (second) letter denotes the states $\left|g^{\prime}\right\rangle$ and $|a\rangle\left(\left|g^{\prime}\right\rangle\right.$ and $\left.|b\rangle\right)$ of system I (system II), and $n$ is the photon number of the cavity mode (Fig. 2). The effective Hamiltonian is then given by $[1,2]$

$$
\begin{aligned}
H_{e f f}= & -\hbar \frac{\Omega_{\mathrm{I}}^{2}}{\Delta_{\mathrm{I}}} \sigma_{g^{\prime} g^{\prime} \mathrm{I}}-\hbar \frac{\mu^{2}}{\Delta_{c}} c^{\dagger} c \sigma_{a a \mathrm{I}}+\hbar \frac{\Omega_{\mathrm{II}}^{2}}{\Delta_{\mathrm{II}}} \sigma_{g^{\prime} g^{\prime} \mathrm{II}} \\
& +\hbar \frac{\mu^{2}}{\Delta_{c}} c^{\dagger} c \sigma_{b b \mathrm{II}}-\hbar \frac{\chi_{\mathrm{I}}^{2}}{\delta} c c^{\dagger} \sigma_{g^{\prime} g^{\prime} \mathrm{I}}+\hbar \frac{\chi_{\mathrm{I}}^{2}}{\delta} c^{\dagger} c \sigma_{a a \mathrm{I}} \\
& +\hbar \frac{\chi_{\mathrm{II}}^{2}}{\delta} c^{\dagger} c \sigma_{g^{\prime} g^{\prime} \mathrm{II}}-\hbar \frac{\chi_{\mathrm{II}}^{2}}{\delta} c c^{\dagger} \sigma_{b b \mathrm{II}} \\
& +\hbar \lambda\left(\left|g^{\prime} g^{\prime}\right\rangle\langle a b|+| a b\rangle\left\langle g^{\prime} g^{\prime}\right|\right),
\end{aligned}
$$

where $\lambda=\chi_{\mathrm{I}} \times \chi_{\mathrm{II}} / \delta$, the last two terms in the second line and the two terms in the third line describe the photon-numberdependent Stark shifts induced by the off-resonant Raman coupling, and the last two terms describe the "dipole" coupling between the two systems (I, II) mediated by the cavity mode and the classical pulses. If the cavity is initially in the vacuum state, then the effective Hamiltonian reduces to

$$
\begin{aligned}
H_{e f f}= & -\hbar\left(\frac{\Omega_{\mathrm{I}}^{2}}{\Delta_{\mathrm{I}}}+\frac{\chi_{\mathrm{I}}^{2}}{\delta}\right) \sigma_{g^{\prime} g^{\prime} \mathrm{I}}+\hbar \frac{\Omega_{\mathrm{II}}^{2}}{\Delta_{\mathrm{II}}} \sigma_{g^{\prime} g^{\prime} \mathrm{II}}-\hbar \frac{\chi_{\mathrm{II}}^{2}}{\delta} \sigma_{b b \mathrm{II}} \\
& +\hbar \lambda\left[\left|g^{\prime} g^{\prime}\right\rangle\langle a b|+| a b\rangle\left\langle g^{\prime} g^{\prime}\right|\right] .
\end{aligned}
$$

Suppose that the two lowest levels $|g\rangle$ and $\left|g^{\prime}\right\rangle$ of each system represent two logical states of a qubit. The time evolution of four logical states for two qubits, under the Hamiltonian (4), are given by

$$
\begin{gathered}
|g\rangle_{\mathrm{I}}|g\rangle_{\mathrm{II}} \rightarrow|g\rangle_{\mathrm{I}}|g\rangle_{\mathrm{II}}, \\
|g\rangle_{\mathrm{I}}\left|g^{\prime}\right\rangle_{\mathrm{II}} \rightarrow e^{-i \epsilon_{\mathrm{II}} t}|g\rangle_{\mathrm{I}}\left|g^{\prime}\right\rangle_{\mathrm{II}}, \\
\left|g^{\prime}\right\rangle_{\mathrm{I}}|g\rangle_{\mathrm{II}} \rightarrow e^{-i \epsilon_{\mathrm{I}} t}\left|g^{\prime}\right\rangle_{\mathrm{I}}|g\rangle_{\mathrm{II}}, \\
\left|g^{\prime}\right\rangle_{\mathrm{I}}\left|g^{\prime}\right\rangle_{\mathrm{II}} \rightarrow e^{-i\left(\epsilon_{\mathrm{I}}+\epsilon_{\mathrm{II}}+\epsilon_{\mathrm{III}}\right) t / 2}\left[\left(\cos \sqrt{\lambda^{2}+\eta^{2}} t\right.\right. \\
\left.+i \frac{\eta}{\sqrt{\lambda^{2}+\eta^{2}}} \sin \sqrt{\lambda^{2}+\eta^{2}} t\right)\left|g^{\prime}\right\rangle_{\mathrm{I}}\left|g^{\prime}\right\rangle_{\mathrm{II}} \\
\left.+i \frac{\lambda}{\sqrt{\lambda^{2}+\eta^{2}}} \sin \sqrt{\lambda^{2}+\eta^{2}} t|a\rangle_{\mathrm{I}}|b\rangle_{\mathrm{II}}\right],
\end{gathered}
$$

where $\epsilon_{\mathrm{I}}=-\Omega_{\mathrm{I}}^{2} / \Delta_{\mathrm{I}}-\chi_{\mathrm{I}}^{2} / \delta, \quad \epsilon_{\mathrm{II}}=\Omega_{\mathrm{II}}^{2} / \Delta_{\mathrm{II}}, \quad \epsilon_{\mathrm{III}}=-\chi_{\mathrm{II}}^{2} / \delta$, and $\eta$ $=\left(\epsilon_{\mathrm{III}}-\epsilon_{\mathrm{I}}-\epsilon_{\mathrm{II}}\right) / 2$. In the case $\epsilon_{\mathrm{I}}+\epsilon_{\mathrm{II}}=\epsilon_{\mathrm{III}}(\eta=0)$, i.e.,

$$
\frac{\Omega_{\mathrm{I}}^{2}}{\Delta_{\mathrm{I}}}+\frac{\chi_{\mathrm{I}}^{2}}{\delta}=\frac{\Omega_{\mathrm{II}}^{2}}{\Delta_{\mathrm{II}}}+\frac{\chi_{\mathrm{II}}^{2}}{\delta},
$$

and by setting $\lambda t=\pi$, we obtain

$$
\begin{gathered}
|g\rangle_{\mathrm{I}}|g\rangle_{\mathrm{II}} \rightarrow|g\rangle_{\mathrm{I}}|g\rangle_{\mathrm{II}}, \\
|g\rangle_{\mathrm{I}}\left|g^{\prime}\right\rangle_{\mathrm{II}} \rightarrow e^{-i \epsilon_{\mathrm{II}} t}|g\rangle_{\mathrm{I}}\left|g^{\prime}\right\rangle_{\mathrm{II}}, \\
\left|g^{\prime}\right\rangle_{\mathrm{I}}|g\rangle_{\mathrm{II}} \rightarrow e^{-i \epsilon \mathrm{I}_{\mathrm{I}} \mid}\left|g^{\prime}\right\rangle_{\mathrm{I}}|g\rangle_{\mathrm{II}}, \\
\left|g^{\prime}\right\rangle_{\mathrm{I}}\left|g^{\prime}\right\rangle_{\mathrm{II}} \rightarrow-e^{-i\left(\epsilon_{\mathrm{I}}+\epsilon_{\mathrm{II}}\right) t}\left|g^{\prime}\right\rangle_{\mathrm{I}}\left|g^{\prime}\right\rangle_{\mathrm{II}} .
\end{gathered}
$$

Then we perform the following one-qubit operations:

$$
\left|g^{\prime}\right\rangle_{\mathrm{I}} \rightarrow e^{i \epsilon_{\mathrm{I}} t}\left|g^{\prime}\right\rangle_{\mathrm{I}},\left|g^{\prime}\right\rangle_{\mathrm{II}} \rightarrow e^{i \epsilon_{\mathrm{II}} t}\left|g^{\prime}\right\rangle_{\mathrm{II}} .
$$

After this, a two-qubit quantum phase gate is realized as follows:

$$
\begin{gathered}
|g\rangle_{\mathrm{I}}|g\rangle_{\mathrm{II}} \rightarrow|g\rangle_{\mathrm{I}}|g\rangle_{\mathrm{II}}, \\
|g\rangle_{\mathrm{I}}\left|g^{\prime}\right\rangle_{\mathrm{II}} \rightarrow|g\rangle_{\mathrm{I}}\left|g^{\prime}\right\rangle_{\mathrm{II}}, \\
\left|g^{\prime}\right\rangle_{\mathrm{I}}|g\rangle_{\mathrm{II}} \rightarrow\left|g^{\prime}\right\rangle_{\mathrm{I}}|g\rangle_{\mathrm{II}}, \\
\left|g^{\prime}\right\rangle_{\mathrm{I}}\left|g^{\prime}\right\rangle_{\mathrm{II}} \rightarrow-\left|g^{\prime}\right\rangle_{\mathrm{I}}\left|g^{\prime}\right\rangle_{\mathrm{II}} .
\end{gathered}
$$

The condition (6) can be met by setting $\Omega_{\mathrm{I}}=\Omega_{\mathrm{II}}$. The reason for this is that due to $\chi_{\mathrm{I}}=\left(\Omega_{\mathrm{I}} \mu / 2\right)\left(1 / \Delta_{c}+1 / \Delta_{\mathrm{I}}\right), \chi_{\mathrm{II}}$ $=\left(\Omega_{\mathrm{II}} \mu 2\right)\left(1 / \Delta_{c}+1 / \Delta_{\mathrm{II}}\right)$, and the above setting $\Delta_{\mathrm{I}}=\Delta_{\mathrm{II}}$, the two effective coupling constants $\chi_{\mathrm{I}}$ and $\chi_{\mathrm{II}}$ in Eq. (6) are identical if the two Rabi frequencies $\Omega_{\mathrm{I}}$ and $\Omega_{\mathrm{II}}$ are set to be the same. It should be mentioned that the setting $\Omega_{\mathrm{I}}=\Omega_{\mathrm{II}}$ can be readily achieved via adjusting the amplitudes of the two pulses.

The energy level diagram can be realized in alkaline-earth atoms or ions using quadrupole allowed transitions between 


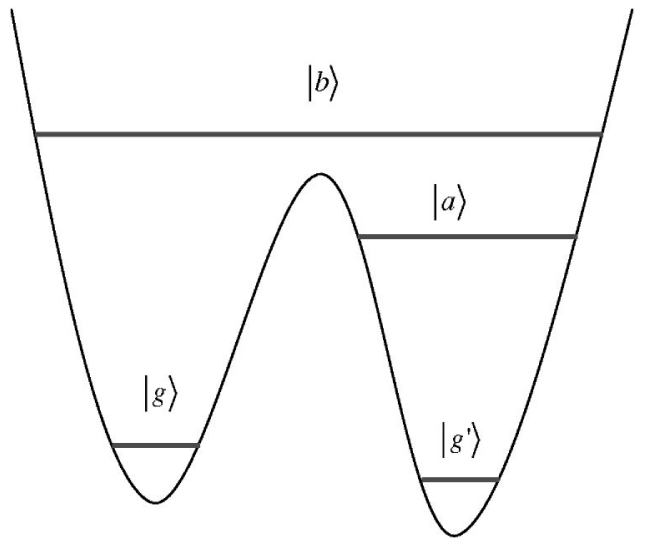

FIG. 3. The first four energy levels of an rf-SQUID with $C$ $=90 \mathrm{fF}, L=100 \mathrm{pH}, \beta_{L}=1.12$, and $\Phi_{x}=0.4995 \Phi_{0}$. The magnetic dipole coupling between the two ground levels $|g\rangle$ and $\left|g^{\prime}\right\rangle$ is much smaller than that between any other two levels, due to the barrier between the two potential wells. The transition frequencies between the excited levels and the ground levels are $\nu_{b g^{\prime}} \sim 24.4 \mathrm{GHz}, \nu_{b g}$ $\sim 21.4 \mathrm{GHz}, \nu_{a g} \sim 16.5 \mathrm{GHz}$, and $\nu_{a g^{\prime}} \sim 19.5 \mathrm{GHz}$, which are much larger than the $|a\rangle \leftrightarrow|b\rangle$ transition frequency $\nu_{a b} \sim 4.9 \mathrm{GHz}$.

metastable $\left|g^{\prime}\right\rangle$ and $|a\rangle$ states. And in many solid-state systems that do not have inversion symmetry the energy level diagram would be generic. For solid-state systems such as quantum dots and superconducting quantum interference devices (SQUIDs), the level structure is straightforward to implement by changing external control parameters (e.g., magnetic flux $\Phi_{x}$ in the case of SQUID qubits) [7,12]. For instance, an rf SQUID with $C=90 \mathrm{fF} L=100 \mathrm{pH}, \beta_{L}=1.12$, and $\Phi_{x}=0.4995 \Phi_{0}$ [13] has the desired level structure as depicted in Fig. 3.

As described above, the controlled phase operation is implemented by choosing $\eta=0$ and carrying out a $2 \pi$ pulse on the $\left|g^{\prime}\right\rangle\left|g^{\prime}\right\rangle$ and $|a\rangle|b\rangle$ transition. During this operation, one excites the states $|a\rangle$ and $|b\rangle$ with a probability $\sin ^{2} \lambda t$ at time $t$. Thus, decoherence rate would be

$$
\gamma_{D}=\frac{1}{T} \int_{0}^{T} \sin ^{2} \lambda t\left(\gamma_{a}+\gamma_{b}\right) d t=\frac{1}{2}\left(\gamma_{a}+\gamma_{b}\right),
$$

where $T=\pi / \lambda ; \gamma_{a}$ and $\gamma_{b}$ are the decay rates of the levels $|a\rangle$ and $|b\rangle$, respectively. We now turn to the experimental matters. First, the typical time $\pi / \lambda$ required for the systemcavity interaction and the decoherence time $\gamma_{D}^{-1}$ need to meet $\pi / \lambda \ll \gamma_{D}^{-1}$, based on which one can easily find that the following relationship between the decoherence rate $\gamma_{D}$ and the coupling strength $\mu$

$$
\mu^{2} \gg \frac{4 \pi \delta \Delta_{c}^{2} \Delta_{\mathrm{I}} \Delta_{\mathrm{II}} \gamma_{D}}{\Omega_{\mathrm{I}} \Omega_{\mathrm{II}}\left(\Delta_{c}+\Delta_{\mathrm{I}}\right)\left(\Delta_{c}+\Delta_{\mathrm{II}}\right)}
$$

should be satisfied. Second, note that the Hamiltonian (2) applies conditional to the adiabatic elimination of the level $|b\rangle$ for system I and the level $|a\rangle$ for system II, thus the occupation probability $P_{b}$ of the level $|b\rangle$ for system I and the occupation probability $P_{a}$ of the level $|a\rangle$ for system II, which are approximately given by

$$
\begin{aligned}
& P_{b} \simeq \frac{1}{2}\left(\frac{4 \Omega_{\mathrm{I}}^{2}}{4 \Omega_{\mathrm{I}}^{2}+\Delta_{\mathrm{I}}^{2}}+\frac{4 \mu^{2}}{4 \mu^{2}+\Delta_{c}^{2}}\right), \\
& P_{a} \simeq \frac{1}{2}\left(\frac{4 \Omega_{\mathrm{II}}^{2}}{4 \Omega_{\mathrm{II}}^{2}+\Delta_{\mathrm{II}}^{2}}+\frac{4 \mu^{2}}{4 \mu^{2}+\Delta_{c}^{2}}\right)
\end{aligned}
$$

(for $\Omega_{\mathrm{I}}, \Omega_{\mathrm{II}}$, and $\mu$ of similar magnitude), need to be negligibly small in order to reduce the gate error. Lastly, the photon lifetime is given by $\kappa^{-1}=Q_{c} / \omega_{c}\left(Q_{c}\right.$ is the quality factor of the cavity) and the cavity has a probability

$$
P_{c} \simeq \frac{1}{2}\left(\frac{4 \chi_{\mathrm{I}}^{2}}{4 \chi_{\mathrm{I}}^{2}+\delta^{2}}+\frac{4 \chi_{\mathrm{II}}^{2}}{4 \chi_{\mathrm{II}}^{2}+\delta^{2}}\right)
$$

of being excited during the operation, thus the effective decay time of the cavity is $\kappa^{-1} P_{c}^{-1}$, which needs to be larger than the system-cavity interaction time $\pi / \lambda$.

As a quantitative example of this technique, consider a SQUID with the parameters given above and with junction's damping resistance $R \sim 1 \mathrm{G} \Omega$. Note that SQUIDs with these parameters are available at the present time [14]. With this choice, the decay time of the levels $|a\rangle$ and $|b\rangle$ would be $\gamma_{a}^{-1} \sim 100 \mu \mathrm{s}$ and $\gamma_{b}^{-1} \sim 40 \mu$ s (i.e., $\gamma_{D}^{-1} \sim 57.1 \mu \mathrm{s}$ ), the $|a\rangle \leftrightarrow|b\rangle$ coupling matrix element is $\phi_{a b} \sim 7.8 \times 10^{-2}$, and the $|a\rangle \leftrightarrow|b\rangle$ transition frequency is $\nu_{a b} \sim 4.9 \mathrm{GHz}$. Hence, we choose $\nu_{c}=\omega_{c} /(2 \pi)=3.6 \mathrm{GHz}$ as the cavity-mode frequency. The SQUID-cavity coupling constant for the $|a\rangle \leftrightarrow|b\rangle$ transition is given by [15] $\mu=(1 / L) \sqrt{\omega_{c} / 2 \mu_{0} \hbar} \phi_{a b} \Phi_{0} \int_{S} \mathbf{B}_{c}(\mathbf{r}) \cdot d \mathbf{S}$, where $S$ is any surface bounded by the SQUID ring and $\mathbf{B}_{c}(\mathbf{r})$ is the magnetic component of the cavity mode in the SQUID loop. For a standing-wave cavity, one has $\mathbf{B}_{c}(z)$ $=\mu_{0} \sqrt{2 / V} \cos \mathrm{k} z$ (k, $V$, and $z$ are the wave number, the cavity volume, and the cavity axis, respectively). For a $10 \times 1$ $\times 1 \mathrm{~mm}^{3}$ standing-wave cavity and a SQUID with a 40 $\times 40 \mu \mathrm{m}^{2}$ loop (located at the cavity-mode antinode), a simple calculation shows $\mu \sim 4.3 \times 10^{8} \mathrm{~s}^{-1}$, i.e., about $0.05 \Delta_{c}$. By choosing $\Delta_{\mathrm{I}}=20 \Omega_{\mathrm{I}}, \Delta_{\mathrm{II}}=20 \Omega_{\mathrm{II}}$, and $\Omega_{\mathrm{I}}=\Omega_{\mathrm{II}}$ $=1.05 \mu$, we have $\delta \sim \mu \sim 20\left(\mu^{2} / \Delta_{c}\right), 20\left(\Omega_{\mathrm{I}, \mathrm{II}}^{2} / \Delta_{\mathrm{I}, \mathrm{II}}\right), 20 \chi_{\mathrm{I}, \mathrm{II}}$. Our calculation shows that (i) the required SQUID-cavity interaction time would be $t_{s-c}=\pi / \lambda \sim 2.8 \mu \mathrm{s}$, much shorter than $\gamma_{D}^{-1}$; (ii) both $P_{a}$ and $P_{b}$ are $\sim 0.01$; (iii) $P_{c} \sim 0.01$, thus the effective decay time of the cavity is $\kappa^{-1} P_{c}^{-1} \sim 44.2 \mu \mathrm{s}$ $\gg t_{s-c}$ for $Q_{c}=10^{4}$, which is realizable since a superconducting cavity with $Q_{c}>10^{5}$ was demonstrated by recent experiments [16]. Therefore, within the present cavity QED technique, the implementation of the proposed scheme is possible with a real physical system.

The method can be applied to any physical systems with a four-level configuration as described above. For different systems, there is no difference to the equations. However, the frequency regimes of the cavity mode are likely to be rather different in the physical realizations with different systems, e.g., optical cavities in the case of atoms while microwave cavities in the case of SQUIDs.

The method can be extended to perform logical operations on many qubits - each qubit is embodied by one of many systems described above inside a cavity, due to long-range coherent interaction between systems mediated via the cavity mode. Full parallel operations are possible (e.g., the two- 
qubit phase gate operation can be performed on each of multi pairs of systems simultaneously), since setting $\delta_{\mathrm{I}}$ and $\delta_{\mathrm{II}}$ of Eq. (2) the same for the two systems in each pair but different for any two pairs, results in that the two systems in each pair couple each other but two different pairs do not couple via the cavity mode.

The experimental challenge is to reduce the gate errors caused due to spontaneous emission from the levels $|a\rangle$ and $|b\rangle$ during the operation. We point out that in this respect our proposal does not offer any advantage. In principle, the gate errors induced due to the decay of the levels $|a\rangle$ and $|b\rangle$ can be greatly reduced as long as the condition $\pi / \lambda \ll \gamma_{D}^{-1}$ is well satisfied. On the other hand, one can check by measurement to be sure that there is no photon population in the cavity or spontaneous emission during the gate performance. If this is done continuously and with sufficient efficiency, this will probably help to avoid the propagation of errors, due to quantum-zeno-type effects [4].
Coupling qubits via the cavity/trap-assisted collision without the excitation of the cavity/vibrational mode was previously reported in Refs. [1,2]. And later this idea was applied to realize a two-qubit phase gate with 3-level atoms/ions, by performing operations beyond two-qubit computational subspace $[5,6]$. However, our purpose is to show that a twoqubit phase gate is achievable with 4-level systems via only two substeps and without real excitation of the cavity mode. We believe that although our scheme is restricted to the 4-level systems, it is of some interest nonetheless, depending on the systems chosen by experimentalists.

This work was partially supported by the National Science Foundation QuBIC program (ECS-0201995), the ITR program (DMR-0325551), and the AFOSR (F49620-01-10439), funded under the Department of Defense University Research Initiative on Nanotechnology (DURINT) Program and by the ARDA.
[1] A. Sørensen and K. Mølmer, Phys. Rev. Lett. 82, 1971 (1999).

[2] S. B. Zheng and G. C. Guo, Phys. Rev. Lett. 85, 2392 (2000).

[3] A. Imamoglu et al., Phys. Rev. Lett. 83, 4204 (1999).

[4] A. Beige, D. Braun, B. Tregenna, and P. L. Knight, Phys. Rev. Lett. 85, 1762 (2000).

[5] X. X. Yi, X. H. Su, and L. You, Phys. Rev. Lett. 90, 097902 (2003)

[6] E. Jane, M. B. Plenio, and D. Jonathan, Phys. Rev. A 65, 050302(R) (2002).

[7] C. P. Yang, S. I. Chu, and S. Han, Phys. Rev. A 67, 042311 (2003).

[8] J. I. Cirac and P. Zoller, Phys. Rev. Lett. 74, 4091 (1995).

[9] S. J. van Enk, J. I. Cirac, and P. Zoller, Phys. Rev. Lett. 79, 5178 (1997).

[10] T. Pellizzari, S. A. Gardiner, J. I. Cirac, and P. Zoller, Phys. Rev. Lett. 75, 3788 (1995).
[11] L. Wang, R. R. Puri, and J. H. Eberly, Phys. Rev. A 46, 7192 (1992).

[12] Prabhakar Pradhan, M. P. Anantram, and Kang L. Wang, e-print quant-ph/0002006.

[13] $C, L$, and $\beta_{L}$ are the SQUID's junction capacitance, loop inductance, and potential shape parameter, respectively. $\Phi_{0}$ $=h / 2 e$ is the flux quantum.

[14] Y. Yu, S. Han, X. Chu, S.-I. Chu, and Z. Wang, Science 296, 889 (2002); I. Chiorescu et al., ibid. 299, 1869 (2003); J. E. Lukens (private communication).

[15] C. P. Yang, S. I. Chu, and S. Han, Phys. Rev. Lett. 92, 117902 (2004).

[16] R. J. Schoelkopf, DURINT annual review meeting, New York City, 2003 (unpublished); P. K. Day, H. G. LeDuc, B. Mazin, A. Vayonakis, and J. Zmuidzinas, Nature (London) 425, 817 (2003). 